\title{
Inferior vena cava filters in the intensive care unit population: single center experience in the united arab emirates
}

\author{
AS Beshyah ${ }^{1 *}$, SA AlBhaisi ${ }^{1}$, K Krishnanreddy $^{2}$, A Khwaja ${ }^{3}$ \\ From ESICM LIVES 2015 \\ Berlin, Germany. 3-7 October 2015
}

\begin{abstract}
Introduction
Pharmacological prophylaxis against venous thromboembolism using low molecular weight heparin (LMWH) has become a standard measure in the intensive care unit (ICU) [1-3]. Risk factors in these patients include critical illness, mechanical ventilation, sedative medications and central venous catheter insertion [2]. In cases where pharmacological prophylaxis is not feasible, inferior vena cava filters (IVCF) have been recommended [1].
\end{abstract}

\section{Objectives}

Evaluation of the indications, course and outcome for filter placement in the critically ill population.

\section{Methods}

We retrospectively reviewed charts of 95 patients who had an IVC filter placed between January 2011 and December 2014 at our institution. We studied the indications for IVCF placement, hospital course, insertion/retrieval dates, contraindications to anticoagulation and the complications associated with the filter. These patients were matched to their appropriate IVC filter guideline indications [4], which were analysed.

\section{Results}

53 of the 95 patients were admitted to ICU with a median age of 47 (20-86) years. Of the total ICU population with placement, 37 (70\%) of the placements were therapeutic and $16(30 \%)$ were prophylactic. In trauma patients $(\mathrm{N}-20), 70 \%$ of the IVC filters were placed as a prophylactic measure whereas in non-traumatic cases $94 \%$ of IVC filters were placed for therapeutic indications. Venous

TSheikh Khalifa Specialty Hospital, Internal Medicine, Abu Dhabi, United Arab Emirates

Full list of author information is available at the end of the article access was mostly via the right internal jugular vein (91\%) and majority were placed infra-renal (96\%). 16 IVC's were retrieved after a median of 67 (21-185) days; representing $30 \%$ of the total and $41 \%$ of the surviving patients. No immediate procedural complications occurred during placement or retrieval; 3 developed DVT and 1 patient developed PE after insertion. Of the total population involved, 14 patients (26\%) died (all being in the nontrauma subgroup). 8 patients were lost to follow up.

\section{Conclusions}

Our review shows that the IVCF practices at our institution are consistent with the accepted recommendations. The vast majority of the patients had a contraindication to anticoagulation therapy. The rate of immediate and delayed complications are low, however further follow up is required to assess the incidence of late complications. All patients who died after placement of an IVC were

\section{ICU IVCF indications (traumatic \& non-traumatic)}

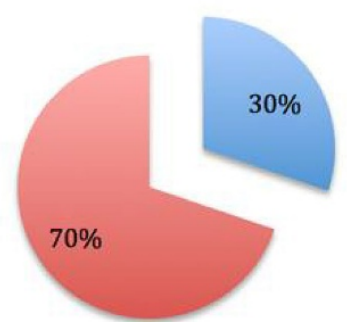

Prophylactic

Therapeutic

Figure $1 \mathrm{ICU}$ indications trauma and non-trauma. 


\section{Therapeutic \& Prophylactic IVCF indications for trauma and non-traumatic patients}

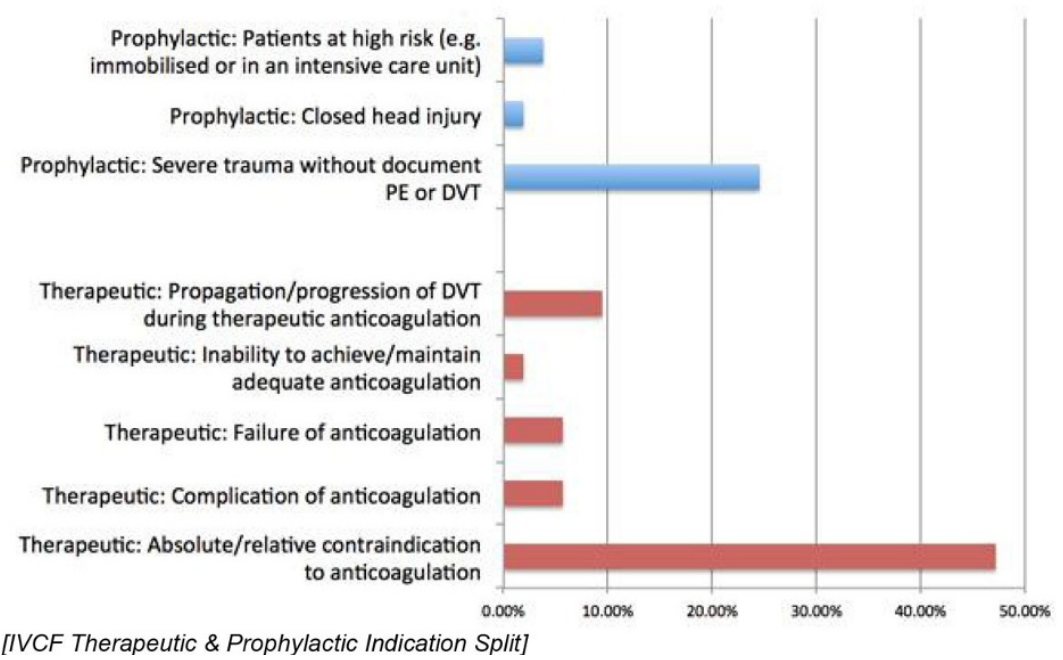

Figure 2 IVCF Therapeutic \& Prophylactic Indication Split.

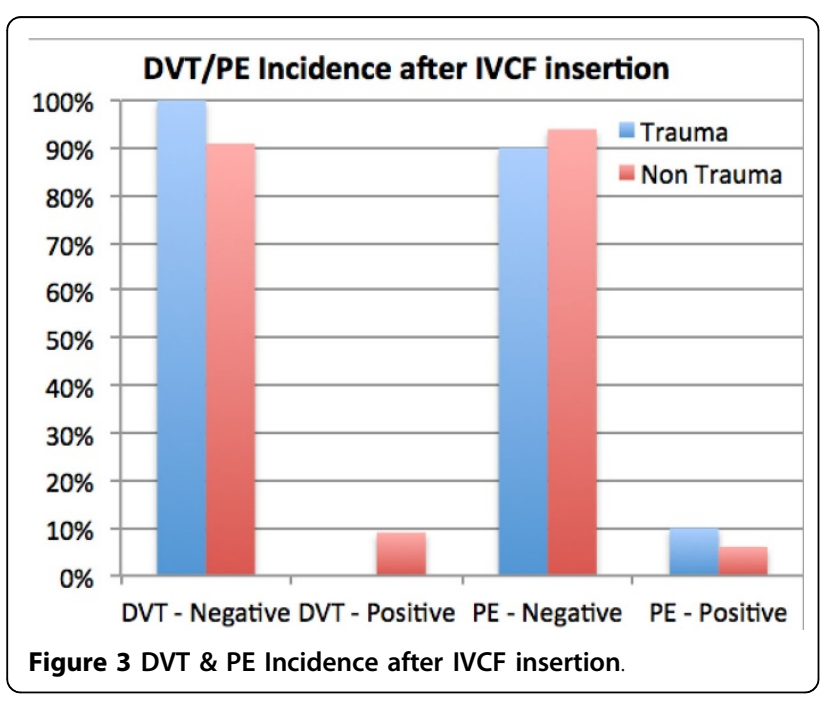

Table 1

\begin{tabular}{ll}
\hline Mean Admission Days (Total population) & 67.4 \\
\hline Shortest Admission (Total population) & 1 \\
\hline Longest Admission (Total population) & 243 \\
\hline Mean Admission Days (Trauma) & 19.1 \\
\hline Shortest Admission (Trauma) & 2 \\
\hline Longest Admission (Trauma) & 76 \\
\hline Mean Admission Days (Non-trauma) & 28.7 \\
\hline Shortest Admission (Non-trauma) & 1 \\
\hline Longest Admission (Non-trauma) & 243 \\
\hline [ICU Admission Data]
\end{tabular}

non-trauma patients with serious co-morbidities, which should allow us to be more liberal in their use in trauma cases.

\section{Authors' details}

'Sheikh Khalifa Specialty Hospital, Internal Medicine, Abu Dhabi, United Arab Emirates. ${ }^{2}$ Sheikh Khalifa Specialty Hospital, Intensive Care Unit, Abu Dhabi, United Arab Emirates. ${ }^{3}$ Sheikh Khalifa Specialty Hospital, Interventional Radiology, Abu Dhabi, United Arab Emirates.

Published: 1 October 2015

\section{References}

1. The intensive care society standards - venous thromboprophylaxis in critical care standards and guidelines.

2. Geerts WSR: Prevention of Venous Thromboembolism in the ICU. Chest 2003, 124:357S-63S.

3. Jain MSG: Venous Thromboembolism and its prevention in critical care. Semin Respir Crit Care Med 1997, 18:79-90.

4. Quality Improvement Guidelines for the Performance of Inferior Vena Cava Filter Placement for the Prevention of Pulmonary Embolism. Drew M. Caplin, et al, for the Society of Interventional Radiology Standards of Practice Committee.

doi:10.1186/2197-425X-3-S1-A970

Cite this article as: Beshyah et al:: Inferior vena cava filters in the intensive care unit population: single center experience in the united arab emirates. Intensive Care Medicine Experimental 2015 3(Suppl 1):A970. 